\title{
Wave propagation in incompressible MHD wave guides: the twisted magnetic Annulus ${ }^{\star}$
}

\author{
R. Erdélyi and B. K. Carter
}

\begin{abstract}
Solar Physics \& upper-Atmosphere Research Group, Department of Applied Mathematics, University of Sheffield, The Hicks Building, Hounsfield Road, Sheffield S3 7RH, UK

e-mail: [robertus;b.carter]@sheffield.ac.uk
\end{abstract}

Received 27 January 2006 / Accepted 2 May 2006

\section{ABSTRACT}

\begin{abstract}
The propagation of MHD waves in a structured magnetic flux tube embedded within a straight vertical magnetic environment is studied analytically. The motivation of this analysis comes from the observations of damped loop oscillations showing that possibly only part of the loop is homogeneous in the radial direction. The general dispersion relation of longitudinal wave propagation is derived for a fully magnetically twisted configuration consisting of a core, annulus and external region each with magnetic field of uniform, yet distinct, twist. Next, a simplified case representing coronal loops is analysed in detail considering magnetic twist just in the annulus, the internal and external regions having straight magnetic field. Modes of oscillations are examined from the general dispersion relation that is suitable for obtaining information on not just oscillations but also on some instability properties of this complex tube structure. It is shown that there are purely surface (i.e. evanescent) and hybrid (spatially oscillatory in the twisted annulus, otherwise evanescent) modes. Except for small wavenumbers, the surface waves show little dispersion; a property making them more suitable for observations. The hybrid modes show a more complex character. Though the frequency range seems to be rather limited, there is a continuum band of frequencies for a given wavenumber. Both short and long wavelength approximations are considered for the symmetrical (sausage) mode and with small twist in particular.
\end{abstract}

Key words. magnetohydrodynamics (MHD) - waves - Sun: oscillations - Sun: magnetic fields

\section{Introduction}

The solar corona is a highly structured and dynamic environment that has captured major interest for many years. The launch of such recent missions as the SOlar and Heliospheric Observatory (SOHO) and Transition Region And Coronal Explorer (TRACE) has delivered a wealth of high cadence data that has allowed a multitude of studies to occur (see e.g. Aschwanden 2004, for an extensive review on observations). A "hot" topic of study has been that of coronal loop oscillations; their cause, their damping, and their role and effect in the age old problem of coronal heating. For a review of oscillations see e.g. Aschwanden (2003), Roberts (2000), Nakariakov \& Roberts (2003) and Wang (2004). The data obtained has allowed the identification of many modes of oscillation including transverse (kink) oscillations (see Aschwanden et al. 1999; Nakariakov et al. 1999; Ofman \& Aschwanden 2002). Standing and propagating kink modes in loops was possibly one of the first successful observations using these high resolution space instruments and the detailed measurements of their amplitude, period, damping times and loop parameters were derived from data. The work and theories developed in this field have led to the evolution of the subjects of coronal seismology (see Roberts 2000; Roberts et al. 1984) and of atmospheric seismology (see de Pontieu et al. 2004, 2005; Erdélyi 2006). Within the solar corona, one specific area of study is that of the oscillations that are observed to occur in plasma magnetically confined to specific configurations, namely coronal loops or flux tubes. Such flux tubes have been studied in

\footnotetext{
* Appendices are only available in electronic form at http://www . edpsciences.org
}

great detail by, among others, Edwin \& Roberts (1983). They find that the existence of inhomogeneities in the form of structuring of the magnetic field enables loops to act as wave guides for a variety of different modes. The typical speeds arising are linked closely to the local Alfvén speed $v_{\mathrm{A}}$, sound speed $c_{\mathrm{S}}$ and tube speed $c_{\mathrm{T}}$. An additional feature of the flux tube is that of twist. Twist is now an accepted concept tied to the existence of coronal loops. Aschwanden (2004) discusses observed noncoplanarity of loops which implies kinked field lines which, in turn, are intimately related to twist. Rotational movement along a loop (as observed by e.g. Chae et al. 2000) also indicates the existence of twist. Besides, it is unfeasible that every flux tube within the solar atmosphere is entirely twist free in spite of the random continuous motions observed at the footpoints. Twisted tubes have been studied in the past by, among others, Dungey \& Loughhead (1954), (Goedbloed 1971a-c, 1983) Parker (1974), Bogdan (1984), Goossens et al. (1995), Klimchuk et al. (2000) but were mainly concerned with stability aspects, the general classification of the MHD spectra or loop geometries. Bennett et al. (1999), however, investigated MHD wave propagation in the case of a uniformly twisted incompressible magnetic flux tube and extended the study of Edwin \& Roberts (1983) by analysing the specific modes that occur in such flux tubes. They found that twist introduces an infinite band of body modes occurring as torsional, or rotational, modes with phase speeds close to the longitudinal Alfvén speed of the loop $c_{\mathrm{A} z}$.

Another topic of much discussion is that of wave damping. All oscillations in the corona are subject to some sort of damping. There are many hypothesised mechanisms by which damping can be explained; sound wave radiation (Stenuit et al. 1998; 
Stenuit et al. 1999), energy absorption due to anomalous viscosity (Nakariakov et al. 1999) and resonant absorption in a thin boundary layer (Ruderman \& Roberts 2002; Aschwanden et al. 2003; Van Doorsselaere et al. 2004). In particular, Ruderman \& Roberts (2002) found that observed damping times would imply an inhomogeneous layer of between $5-45 \%$ of the tube's radius. These studies led us (Carter \& Erdélyi 2004; Carter 2005) and Mikhalyaev \& Solov'ev (2005) to the concept of MHD wave studies in a flux tube model consisting of a cord surrounded by a magnetic shell. Carter \& Erdélyi (2004) and Carter (2005) investigated the case of a straight magnetic core and external field and a twisted magnetic annulus. The general dispersion relation was found and cases of long and short wavelength briefly considered. In this paper we give a more comprehensive analysis and consider some specific applications to the solar atmosphere. Mikhalyaev \& Solov'ev (2005), who considered the case of a straight magnetic flux tube consisting of a core, shell and an external magnetic field of different strengths, analysed the specific modes supported by this configuration and found a variety of modes occurring. Two slow and two fast modes were found supported by the cord (oscillating in phase) and the shell (oscillations with opposite phase). In the present work we extend the current ideas to a magnetic configuration consisting of a magnetic core, shell and external ambient field all with an applied twist. We model the predicted inhomogeneous layer as a twisted annulus embedded within a straight magnetic field. We neglect the effect of gravity, the emphasis being on the role of the magnetic field. We find the general dispersion relation for the case of twist in each region and for the case of a straight core and external field for degenerated magnetosonic waves (of course, in incompressible plasma, the sound speed is infinite and appropriate limits must be taken as it will be shown in the paper). The general dispersion relation is solved analytically for the sausage $(m=0)$ case and in the thin tube (long wavelength) and short wavelength approximations considering further thin annulus and small twist approximation. This configuration can support various modes. For incompressible sausage modes it is found that only surface waves are supported in the internal and external regions with both body (i.e. hybrid) and surface modes occurring in the twisted annulus. It is the absence of twist combined with the assumption of incompressibility that restricts to solely surface modes.

\section{Governing equations}

Let us restrict the investigation to an incompressible plasma for which fast waves are removed from the system and Alfvén and slow waves merge. The modes of oscillation in a twisted magnetic annulus with straight internal and external magnetic field will be studied. To do so we first suppose, in cylindrical polar coordinates, magnetic field of the form

$\boldsymbol{B}=\left(0, B_{\theta}(r), B_{z}(r)\right)$,

embedded in an incompressible plasma. We take the plasma density $\rho$ as uniform and the field and plasma pressure $p(r)$ structured in the radial direction so as to satisfy the pressure balance

$\frac{\mathrm{d}}{\mathrm{d} r}\left(p+\frac{B^{2}}{2 \mu}\right)=\frac{-B_{\theta}^{2}}{r \mu}$.

Here $\mu$ is the magnetic permeability and $B(r)=\left(B_{\theta}^{2}+B_{z}^{2}\right)^{1 / 2}=|\boldsymbol{B}|$ denotes the equilibrium magnetic field strength.

\subsection{MHD equations}

Let $\boldsymbol{b}$ denote a perturbation in the magnetic field. The MHD equations, linearised about an equilibrium given by (2) for an incompressible plasma, as found for example in Bennett et al. (1998) can then be written:

$$
\begin{aligned}
\rho \frac{\partial^{2} \xi}{\partial t^{2}} & =-\nabla p_{\mathrm{T}}+\frac{1}{\mu}(\boldsymbol{B} \cdot \nabla) \boldsymbol{b}+\frac{1}{\mu}(\boldsymbol{b} \cdot \nabla) \boldsymbol{B}, \\
\boldsymbol{b} & =(\boldsymbol{B} \cdot \nabla) \xi-(\xi \cdot \nabla) \boldsymbol{B} \\
\nabla \cdot \xi & =0
\end{aligned}
$$

where $\xi$ is the Lagrangian displacement vector and

$p_{\mathrm{T}}=p+\boldsymbol{B} \cdot \boldsymbol{b} / \mu$

is the total pressure perturbation for kinetic plasma pressure $p$ and magnetic field perturbation $\boldsymbol{b}$. The magnetic perturbation can then be eliminated from Eqs. (3), (4) and we can rewrite Eqs. (3)-(6) in component form for the Lagrangian displacement:

$$
\begin{aligned}
& \mathcal{D} \xi_{r}=\frac{\partial p_{\mathrm{T}}}{\partial r}+\frac{2 B_{\theta}}{\mu r}(\boldsymbol{B} \cdot \nabla) \xi_{\theta}-\frac{2 B_{\theta}}{\mu} \frac{\mathrm{d}}{\mathrm{d} r}\left(\frac{B_{\theta}}{r}\right) \xi_{r} \\
& \mathcal{D} \xi_{\theta}=\frac{1}{r} \frac{\partial p_{\mathrm{T}}}{\partial \theta}-\frac{2 B_{\theta}}{\mu r}(\boldsymbol{B} \cdot \nabla) \xi_{r} \\
& \mathcal{D} \xi_{z}=\frac{\partial p_{\mathrm{T}}}{\partial z}
\end{aligned}
$$

where

$$
\frac{\partial}{\partial r}\left(r \xi_{r}\right)+\frac{\partial \xi_{\theta}}{\partial \theta}+r \frac{\partial \xi_{z}}{\partial z}=0,
$$

and the operator $\mathcal{D}$ is

$\mathcal{D}=\frac{1}{\mu}(\boldsymbol{B} \cdot \nabla)^{2}-\rho \frac{\partial^{2}}{\partial t^{2}}$

Let us Fourier analyse the system

$f \rightarrow f(r) \mathrm{e}^{\mathrm{i}\left(m \theta+k_{z} z-\omega t\right)}$,

where $m(=0, \pm 1, \pm 2, \ldots)$ is the azimuthal order of the mode, $k_{z}$ is the longitudinal wavenumber, and $\omega$ is the frequency of the mode. It is now possible to combine Eqs. (7)-(10) and return two first order coupled differential equations for, say, the radial component of the Lagrangian displacement $\xi_{r}$ and total pressure perturbation $p_{\mathrm{T}}$ :

$D \frac{\mathrm{d}}{\mathrm{d} r}\left(r \xi_{r}\right)=C_{1} r \xi_{r}-C_{2} r p_{\mathrm{T}}$,
$D \frac{\mathrm{d} p_{\mathrm{T}}}{\mathrm{d} r}=C_{3} \xi_{r}-C_{1} p_{\mathrm{T}}$

where

$D=\rho\left(\omega^{2}-\omega_{\mathrm{A}}^{2}\right)$,

$C_{1}=-\frac{2 m B_{\theta}}{\mu r^{2}}\left(\frac{m}{r} B_{\theta}+k_{z} B_{z}\right)$,

$C_{2}=-\left(\frac{m^{2}}{r^{2}}+k_{z}^{2}\right)$

$C_{3}=D^{2}+D \frac{2 B_{\theta}}{\mu} \frac{\mathrm{d}}{\mathrm{d} r}\left(\frac{B_{\theta}}{r}\right)-\frac{4 B_{\theta}^{2}}{\mu r^{2}} \rho \omega_{\mathrm{A}}^{2}$, 
for which $\omega_{\mathrm{A}}$ is the Alfvén frequency defined as

$\omega_{\mathrm{A}}=\frac{1}{(\mu \rho)^{1 / 2}}\left(\frac{m}{r} B_{\theta}+k_{z} B_{z}\right)$.

Equations (13) and (14) are a special (incompressible) case of the compressible equations found by, amongst others, Appert et al. (1974), Goedbloed (1971a) and Hain \& Lüst (1958). It is now possible to eliminate $\xi_{r}$ from Eqs. (13) and (14) to leave a second order differential equation solely in terms of $p_{\mathrm{T}}$ :

$$
\begin{aligned}
\frac{\mathrm{d}^{2} p_{\mathrm{T}}}{\mathrm{d} r^{2}} & +\left\{\frac{C_{3}}{r D} \frac{\mathrm{d}}{\mathrm{d} r}\left(\frac{r D}{C_{3}}\right)\right\} \frac{\mathrm{d} p_{\mathrm{T}}}{\mathrm{d} r} \\
& +\left\{\frac{C_{3}}{r D} \frac{\mathrm{d}}{\mathrm{d} r}\left(\frac{r C_{1}}{C_{3}}\right)+\frac{1}{D^{2}}\left(C_{2} C_{3}-C_{1}^{2}\right)\right\} p_{\mathrm{T}}=0 .
\end{aligned}
$$

In general Eq. (15) is a variable coefficient ODE that can only be solved analytically under special circumstances.

\section{The dispersion relation}

For simplicity we consider the case for which all three regions have constant longitudinal field and uniform twist such that $B_{\theta}(r) / r$ and $B_{z}(r)$ are constant. We consider such a field and using the notation

$\boldsymbol{B}= \begin{cases}\boldsymbol{B}_{\mathrm{i}}=\left(0, A_{\mathrm{i}} r, B_{\mathrm{i}}\right), & r<a, \\ \boldsymbol{B}_{0}=\left(0, A_{0} r, B_{0}\right), & a<r<R, \\ \boldsymbol{B}_{\mathrm{e}}=\left(0, A_{\mathrm{e}} r, B_{\mathrm{e}}\right), & r>R,\end{cases}$

where $A_{\mathrm{i}}, A_{0}, A_{\mathrm{e}}, B_{\mathrm{i}}, B_{0}$, and $B_{\mathrm{e}}$ are constant. The densities of the internal, annulus and external regions are denoted as $\rho_{\mathrm{i}}, \rho_{0}$ and $\rho_{\mathrm{e}}$, respectively. This configuration reduces the Alfvén frequency $\omega_{\mathrm{A}}$ and the coefficients $D, r C_{1}$ and $C_{3}$ to constants:

$\begin{array}{ll}\omega_{\mathrm{A}}=\frac{1}{(\mu \rho)^{1 / 2}}\left(m A+k_{z} B_{0}\right), & D=\rho\left(\omega^{2}-\omega_{\mathrm{A}}^{2}\right), \\ C_{1}=-\frac{2 m A}{\mu r}\left(m A+k_{z} B_{0}\right), & C_{3}=D^{2}-\frac{4 A^{2}}{\mu} \rho \omega_{\mathrm{A}}^{2} .\end{array}$

With this simplification one can recover a second order differential equation in $p_{\mathrm{T}}$ :

$\frac{\mathrm{d}^{2} p_{\mathrm{T}}}{\mathrm{d} r^{2}}+\frac{1}{r} \frac{\mathrm{d} p_{\mathrm{T}}}{\mathrm{d} r}-\left(\frac{m^{2}}{r^{2}}+m_{0}^{2}\right) p_{\mathrm{T}}=0$,

where

$m_{0}^{2}=k_{z}^{2}\left(1-\frac{4 A_{0}^{2} \omega_{\mathrm{A} 0}^{2}}{\mu \rho_{0}\left(\omega^{2}-\omega_{\mathrm{A} 0}^{2}\right)^{2}}\right)$,

in which 0 subscripts (which correspond to the annulus) are replaced by $i, e$ corresponding to the internal and external regions, respectively. Equation (19) is the modified Bessel's equation with solutions the modified Bessel functions $I_{m}\left(m_{0} r\right), K_{m}\left(m_{0} r\right)$, of the first and second kind, respectively. For $m_{0}^{2}<0$, Eq. (19) becomes Bessel's equation with solutions the Bessel functions of the first and second kind, $J_{m}\left(m_{0} r\right)$ and $Y_{m}\left(m_{0} r\right)$. Appendix A contains the full dispersion relation for this general fully twisted configuration for arbitrary poloidal wavenumber, $m$. Included is the detailed analysis of reducing this dispersion relation to the dispersion relation for the simpler case of a straight magnetic internal and external region while maintaining the uniform twist within the annulus.

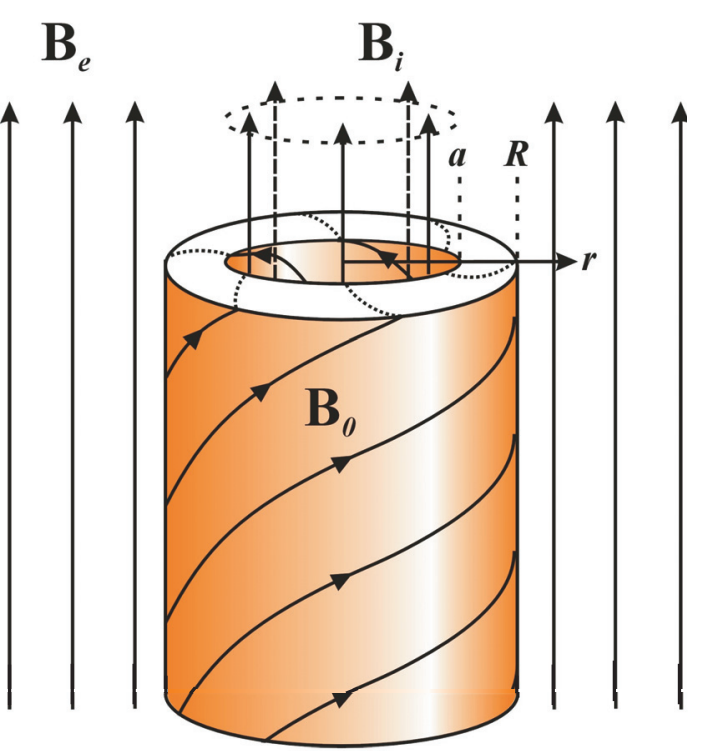

Fig. 1. The equilibrium configuration for a straight magnetic core and a twisted magnetic annulus embedded in a straight ambient magnetic field.

\subsection{Straight magnetic cord surrounded by twisted magnetic annulus}

We consider, specifically, a flux tube consisting of a twisted magnetic annulus embedded in a straight magnetic field, as in Fig. 1 given by:

$\boldsymbol{B}= \begin{cases}\boldsymbol{B}_{\mathrm{i}}=\left(0,0, B_{\mathrm{i}}\right), & r<a, \\ \boldsymbol{B}_{0}=\left(0, A_{0} r, B_{0}\right), & a<r<R, \\ \boldsymbol{B}_{\mathrm{e}}=\left(0,0, B_{\mathrm{e}}\right), & r>R,\end{cases}$

in which the constants $A_{\mathrm{i}}, A_{\mathrm{e}}$ from configuration (16) are set to 0 . For the configuration described by Eqs. (21), the governing Eq. (19) has solutions like $p_{\mathrm{T}} \sim I_{m}\left(m_{0} r\right)$ and $\sim K_{m}\left(m_{0} r\right)$, the modified Bessel functions of order $m$. We require that $p_{\mathrm{T}}$ is finite at $r=0$ giving

$p_{\mathrm{T}}=\alpha I_{m}\left(m_{\mathrm{i}} r\right), \quad r<a$,

and we require that the solutions are evanescent as $r \rightarrow \infty$ giving

$p_{\mathrm{T}}=\delta K_{m}\left(m_{\mathrm{e}} r\right), \quad r>R$

for $\alpha, \delta$ arbitrary constants. Since the magnetic field has no twist in these two regions we notice that $m_{\mathrm{i}}$ and $m_{\mathrm{e}}$ reduce to $\left|k_{z}\right|$, meaning $m_{\mathrm{i}}^{2}$ and $m_{\mathrm{e}}^{2}$ are always positive. However, within the annulus $m_{0}$ may be real or purely imaginary. For frequencies that satisfy

$\omega_{\mathrm{A} 0}^{2}-\frac{2 A_{0} \omega_{\mathrm{A} 0}}{\sqrt{\mu \rho_{0}}}<\omega^{2}<\omega_{\mathrm{A} 0}^{2}+\frac{2 A_{0} \omega_{\mathrm{A} 0}}{\sqrt{\mu \rho_{0}}}$

$m_{0}^{2}$ is indeed negative and so body modes $\left(J_{m}, Y_{m}\right.$ solutions of the Bessel equation) are found to occur. In this latter case it is convenient to write

$n_{0}^{2}=-m_{0}^{2}>0$, 


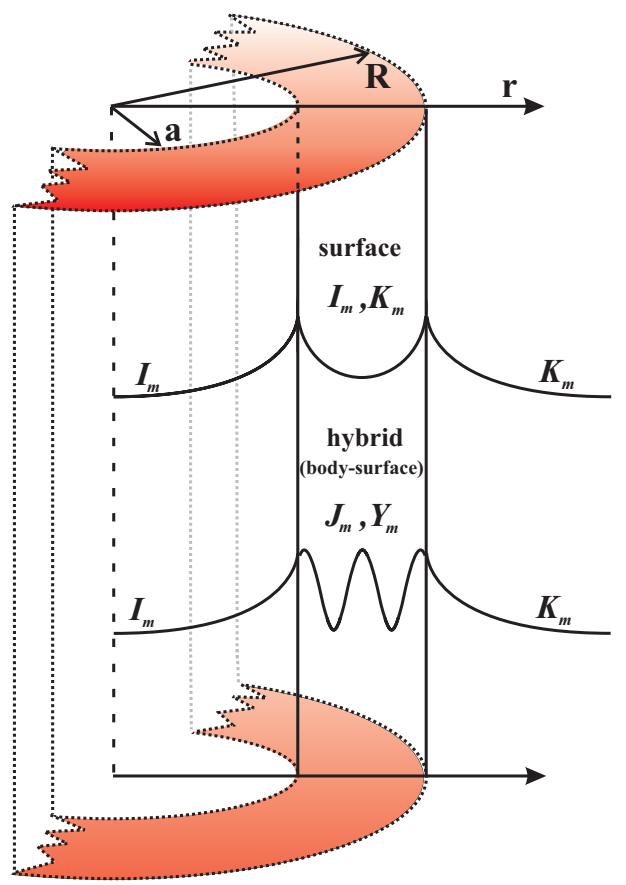

Fig. 2. Body and surface waves determined by the value of $m_{0}^{2}$ in each region. The modified Bessel functions $I_{m}$ and $K_{m}$ for $m_{0}^{2}>0$ giving surface waves and Bessel's equation when $m_{0}^{2}<0$ with solutions the Bessel functions, $J_{m}, Y_{m}$ of the first and second kind resulting in body waves.

and then the solution to Eq. (19) within the annulus may be written in general as

$\begin{array}{ll}p_{\mathrm{T}}=\beta I_{m}\left(m_{0} r\right)+\gamma K_{m}\left(m_{0} r\right), & m_{0}^{2}>0, \\ p_{\mathrm{T}}=\beta J_{m}\left(n_{0} r\right)+\gamma Y_{m}\left(n_{0} r\right), & m_{0}^{2}<0 .\end{array}$

This allows two different mode configurations to occur. The case of $m_{0}^{2}>0$ results in the waves everywhere being evanescent surface waves whereas in the latter case, $m_{0}^{2}<0$, we find the solution is in the form of hybrid modes; surface in the internal and external regions and body in the annulus. These are shown schematically in Fig. 2.

For the boundary conditions we require the conservation of total Lagrangian pressure across the perturbed boundaries and that the normal perturbation $\xi_{r}$ remains continuous across the boundaries $r=a$ and $r=R$ :

$\left[\xi_{r}\right]_{r=a+\varepsilon}^{r=a-\varepsilon}=\left[\xi_{r}\right]_{r=R+\varepsilon}^{r=R-\varepsilon}=0$

$\left[p_{\mathrm{T}}-\frac{B_{\theta}^{2}}{\mu r} \xi_{r}\right]_{r=a+\varepsilon}^{r=a-\varepsilon}=\left[p_{\mathrm{T}}-\frac{B_{\theta}^{2}}{\mu r} \xi_{r}\right]_{r=R+\varepsilon}^{r=R-\varepsilon}=0$.

These boundary conditions Eqs. (26) and (27), when applied to solutions for $p_{\mathrm{T}}$ after some cumbersome algebra and eliminating $\xi_{r}$, yield the general dispersion relation for the more general configuration (i.e. twisted core, annulus and external field) defined in Eq. (16). Appendix A gives the full general dispersion relation for a uniform, yet distinct, twist in all three regions, namely the core, the annulus and the external magnetic field. It also outlines the steps involved in reducing this dispersion relation to a more specific case by setting $A_{\mathrm{i}}=0$ and $A_{\mathrm{e}}=0$, i.e. no twist in the core and ambient external region. It is possible to express this dispersion relation in terms of the dispersion relation found by Bennett et al. (1999). Appendix B shows the dispersion relation Eq. (28a) written as the dispersion relation from Bennett et al. (1999) plus terms due to the annulus. We obtain the dispersion relation for a uniformly twisted annulus embedded in a straight magnetic field:

$$
\begin{aligned}
& \frac{\Xi_{a K}-\Xi_{\mathrm{i}}+\Xi_{a K} \Xi_{\mathrm{i}} \frac{A_{0}^{2}}{\mu}}{\Xi_{a I}-\Xi_{\mathrm{i}}+\Xi_{a I} \Xi_{\mathrm{i}} \frac{A_{0}^{2}}{\mu}} \frac{K_{m}\left(m_{0} a\right)}{I_{m}\left(m_{0} a\right)}= \\
& \frac{K_{m}\left(m_{0} R\right)}{I_{m}\left(m_{0} R\right)} \frac{\Xi_{R K}-\Xi_{\mathrm{e}}+\Xi_{R K} \Xi_{\mathrm{e}} \frac{A_{0}^{2}}{\mu}}{\Xi_{R I}-\Xi_{\mathrm{e}}+\Xi_{R I} \Xi_{\mathrm{e}} \frac{A_{0}^{2}}{\mu}}, \quad m_{0}^{2}>0,
\end{aligned}
$$

for purely surface waves, and

$$
\begin{aligned}
& \frac{\Xi_{a Y}-\Xi_{\mathrm{i}}+\Xi_{a Y} \Xi_{\mathrm{i}} \frac{A_{0}^{2}}{\mu}}{\Xi_{a J}-\Xi_{\mathrm{i}}+\Xi_{a J} \Xi_{\mathrm{i}} \frac{A_{0}^{2}}{\mu}} \frac{Y_{m}\left(n_{0} a\right)}{J_{m}\left(n_{0} a\right)}= \\
& \frac{Y_{m}\left(n_{0} R\right)}{J_{m}\left(n_{0} R\right)} \frac{\Xi_{R Y}-\Xi_{\mathrm{e}}+\Xi_{R Y} \Xi_{\mathrm{e}} \frac{A_{0}^{2}}{\mu}}{\Xi_{R J}-\Xi_{\mathrm{e}}+\Xi_{R J} \Xi_{\mathrm{e}} \frac{\mathrm{A}_{0}^{2}}{\mu}}, \quad m_{0}^{2}<0,
\end{aligned}
$$

for hybrid (surface-body) waves. Here

$$
\Xi_{\alpha X}=\frac{\left(\omega^{2}-\omega_{\mathrm{A} 0}^{2}\right) \frac{m_{0} \alpha X_{m}^{\prime}\left(m_{0} \alpha\right)}{X_{m}\left(m_{0} \alpha\right)}-\frac{2 m A_{0} \omega_{\mathrm{A} 0}}{\sqrt{\mu \rho_{0}}}}{\rho_{0}\left(\omega^{2}-\omega_{\mathrm{A} 0}^{2}\right)^{2}-\frac{4 A_{0}^{2} \omega_{\mathrm{A} 0}^{2}}{\mu}},
$$

where $X$ denotes the corresponding Bessel function $J, Y$ or modified Bessel function $I, K$, and $\alpha$ is replaced by $a, R$ for the corresponding region,

$$
\begin{aligned}
\Xi_{\mathrm{i}} & =\frac{|k| a I_{m}^{\prime}(|k| a)}{\rho_{\mathrm{i}}\left(\omega^{2}-\omega_{\mathrm{Ai}}^{2}\right) I_{m}(|k| a)}, \\
\Xi_{\mathrm{e}} & =\frac{|k| R K_{m}^{\prime}(|k| R)}{\rho_{\mathrm{e}}\left(\omega^{2}-\omega_{\mathrm{Ae}}^{2}\right) K_{m}(|k| R)}, \\
m_{0}^{2} & =k_{z}^{2}\left(1-\frac{4 A_{0}^{2} \omega_{\mathrm{A} 0}^{2}}{\mu \rho_{0}\left(\omega^{2}-\omega_{\mathrm{A} 0}^{2}\right)^{2}}\right)=-n_{0}^{2},
\end{aligned}
$$

where the dash' denotes the derivative with respect to the argument of the Bessel functions. The Alfvén frequencies in the annulus, internal and external regions are given by $\omega_{\mathrm{A} 0}, \omega_{\mathrm{Ai}}$, and $\omega_{\mathrm{Ae}}$, respectively such that

$\omega_{\mathrm{A} 0}=\frac{1}{\sqrt{\mu \rho_{0}}}\left(m A_{0}+k_{z} B_{0}\right)$

$\omega_{\mathrm{Ai}}=\frac{k_{z} B_{\mathrm{i}}}{\sqrt{\mu \rho_{\mathrm{i}}}}, \quad \omega_{\mathrm{Ae}}=\frac{k_{z} B_{\mathrm{e}}}{\sqrt{\mu \rho_{\mathrm{e}}}}$.

Equations (28a) and (28b) are the general dispersion relations for an incompressible twisted magnetic annulus embedded in an untwisted environment. In the limit of no core, when $a \rightarrow 0$, we recover the dispersion relation from Bennett et al. (1999) for the case of a uniformly twisted incompressible magnetic tube, and further, setting $A_{0}=0$, we recover the case for a straight magnetic tube as in Edwin \& Roberts (1983).

\section{Applications to solar structures}

Let us now consider three specific cases to study the array of modes given by Eqs. (28a) and (28b). We examine the effect of varying annulus width and the effect of applying different degrees of twist on the propagating sausage $(m=0)$ modes. 

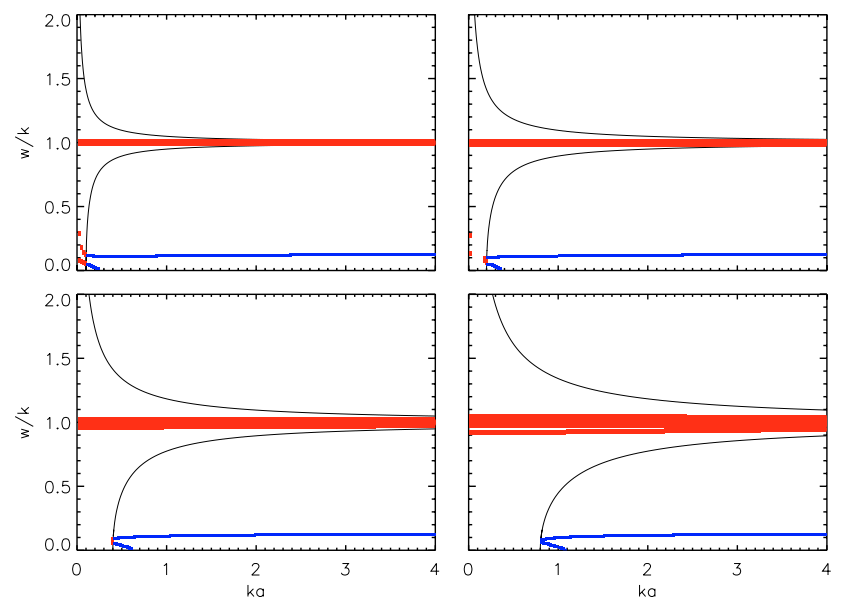

Fig. 3. Phase velocity of the $m=0$ sausage modes determined from Eqs. (28a), (28b) for a twisted annulus embedded in a straight internal, $B_{\mathrm{i}}=B_{0}$, and external, $B_{\mathrm{e}}=0.1 B_{0}$, magnetic field with $a / R=0.80$, $\rho_{0} / \rho_{\mathrm{i}}=1, \rho_{0} / \rho_{\mathrm{e}}=1$. Shown are plots for increasing twists $B_{\theta} / B_{z}=$ $0.05,0.1,0.2,0.4$ v's normalised phase speed $c_{\mathrm{ph}} / c_{\mathrm{Az}}$. Also shown is the envelope separating the hybrid (dotted) modes, as an infinite band of eigensolutions in the neighbourhood of $c_{\mathrm{A} z}$, and the surface modes.
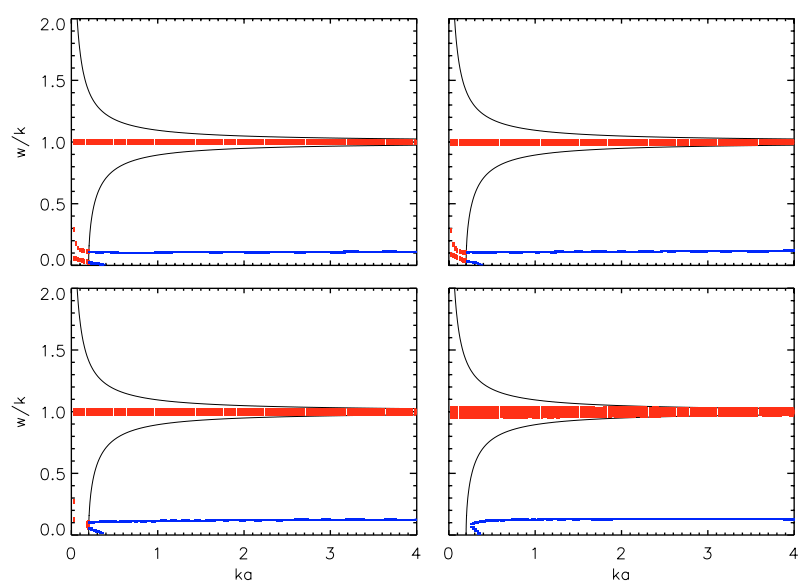

Fig. 4. Same as Fig. 3 but with fixed twist $B_{\theta} / B_{z}=0.1$. Shown are plots for annulus width $5 \%, 10 \%, 20 \%$ and $40 \%$ of the tubes radius.

\subsection{Photospheric tubes}

Photospheric phenomena such as sunspots, plage regions, largescale unipolar areas and supergranulation fields at supergranule boundaries all contain a much higher concentration of magnetic field within the tubes than in the surrounding atmosphere (Aschwanden 2004). Figures 3 and 4 show the solutions of the dispersion relations of the hybrid and surface modes under expected typical photospheric tube conditions. (Note, however, that stratification effects have been ignored which could be relevant under photospheric conditions.) We model numerically the conditions as a magnetic intensity enhancement within the tube with the longitudinal magnetic field strength in the internal and annulus regions being 10 or 20 times that of the external field. Strictly speaking the ambient field for a photospheric tube should be considered vanishing, but the current choice of parameters will give a very good approximation. It also helps to keep the numerical complications to a minimum. When the magnetic twist is increased, the hybrid modes cover a wider range of phase speeds, centered around the annulus' longitudinal Alfvén speed $c_{\mathrm{Az}}$. Notice the avoided crossing feature in the two modes with phase speeds between $c_{\mathrm{ph}} \approx 0$ and 0.1 in e.g. Fig. 4 . They appear

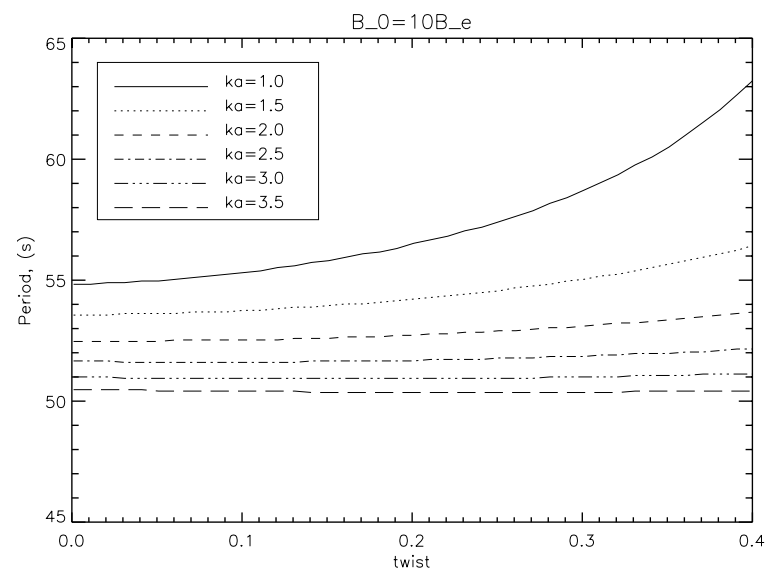

Fig. 5. This plot shows the effect of $B_{\theta}$ on the periods of oscillation for parameters the same as in Fig. 3 for the surface mode occurring at a phase speed between 0.09 and 0.11 for a series of wavelengths $(k a=1.0,1.5,2.0,2.5,3.0,3.5)$.

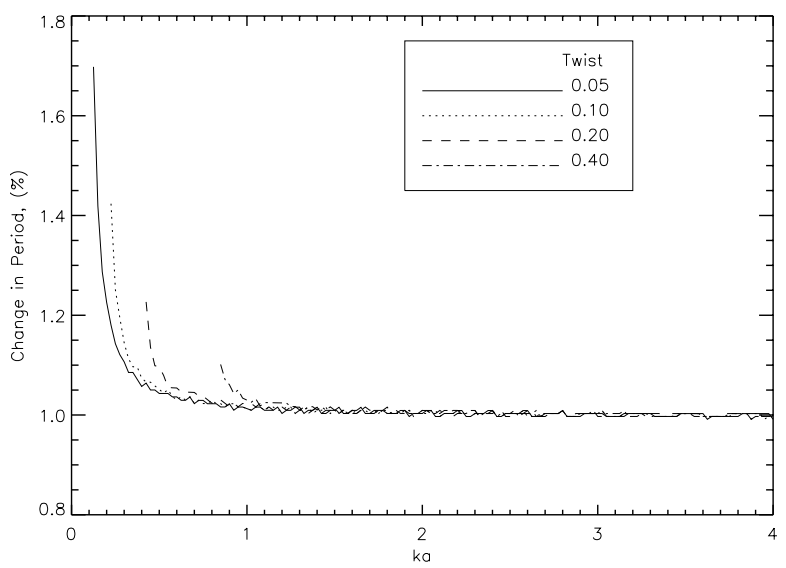

Fig. 6. Percentage difference between a tube magnetic field enhancement of $B_{0} / B_{\mathrm{e}}=20$ compared to $B_{0} / B_{\mathrm{e}}=10$ for a twist of $B_{\theta} / B_{0}=0.1$ of the surface wave in Fig. 3 at a phase speed of $\approx 0.1$.

more pronounced as twist is made large. When the annulus width is increased, the infinite band of hybrid modes broadens slightly but seems to have no other significant effect. Also plotted, in Fig. 5, is the effect of increasing the twist on the phase speed of the surface mode seen in Fig. 3 occurring with phase speed $\approx 0.1$. Parameters are taken the same as those used in Fig. 3. It is clear from this plot that increasing the strength of twist in the annulus has a more significant effect on the phase speed for longer wavelengths. For $k a$ values over around 3, increasing the twist seems to have little or no effect.

Figure 6 shows the change in period, as a percentage, from a tube magnetic field enhancement of 10 to 20 times the external field. When the value of $k a$ is larger the period changes by $\approx 1 \%$ independent of the degree of twist. For longer wavelengths however, increasing the magnetic field enhancement has more effect on the value of the period of oscillation. For a magnetic twist of 0.05 , reducing $k a$ from 0.4 to 0.2 (long wavelength) gives a rise in percentage form $1.0 \%$ to $1.2 \%$. However, for shorter wavelengths, reducing $k a$ from e.g. 2.2 to 2.0 , there is no change from a $1 \%$ increase in period. 

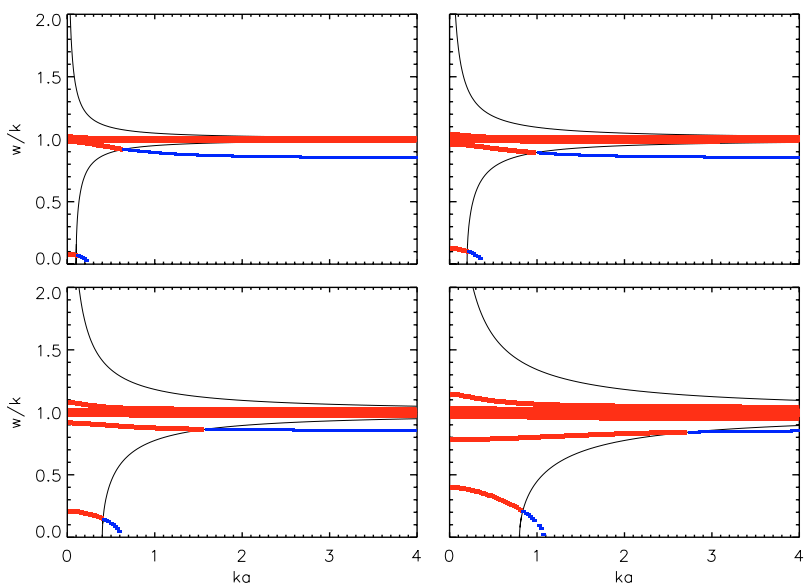

Fig. 7. Same as Fig. 3 but with parameters approximating a dense tube in a magnetic environment. $B_{\mathrm{i}}=B_{0}, B_{\mathrm{e}}=0.5 B_{0}, a / R=0.80, \rho_{0} / \rho_{\mathrm{i}}=1$, $\rho_{0} / \rho_{\mathrm{e}}=2$. Shown are plots for twist $B_{\theta} / B_{z}=0.05,0.1,0.2,0.4$.
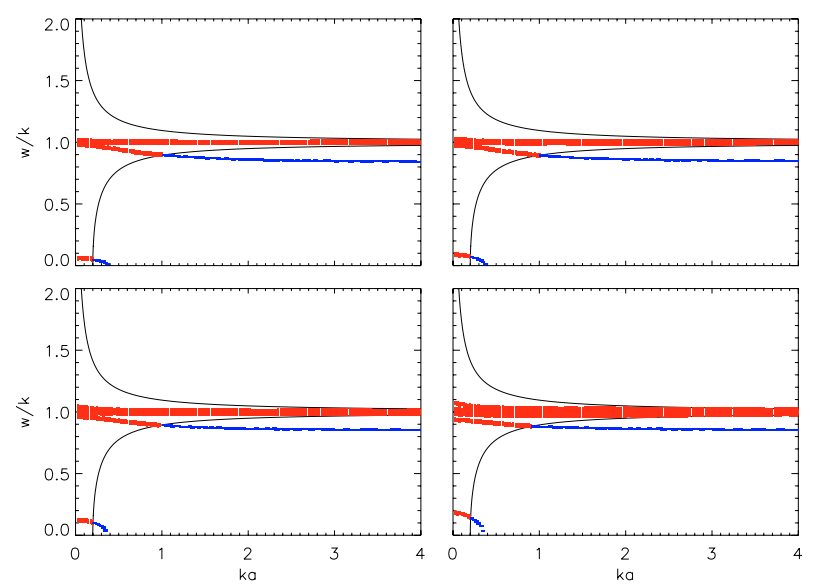

Fig. 8. Same as Fig. 7 but with fixed twist $B_{\theta} / B_{z}=0.1$. Shown are plots for annulus width $5 \%, 10 \%, 20 \%$ and $40 \%$ of the tubes radius.

\subsection{Dense flux tubes in a magnetic environment}

Figures 7 and 8 illustrate the behaviour of the modes in parameters approximating a dense tube. We model this as a density enhancement within the tube taking the external ambient magnetic field as half that of the longitudinal components of the internal and annulus fields. We approximate the (internal and annulus) tube density of twice that in the external region. We found, for a fixed annulus thickness of $20 \%$ of the radius of the tube and fixed strength of the longitudinal components of the magnetic field in each region (Fig. 7) the increase in width of the infinite band of hybrid modes as the twist is increased. Also observed is the introduction of a mode when twist is introduced to the annulus and the increase in phase speed of this mode as the applied twist is made larger. A similar effect occurs as the annulus thickness, for fixed twist (Fig. 8), is increased. It is, however, less pronounced than the varying twist case. Figure 9 shows the change in period of the surface mode in Fig. 7 as twist is increased. Note that for lower $k a$ values the period is less for smaller twist but this changes as twist becomes larger.

\subsection{Evacuated tubes}

Evacuated tubes (where the density in the tube is less than the external density) are now modelled and the behaviour of the waves are shown in Figs. 10 and 11. We model an evacuated

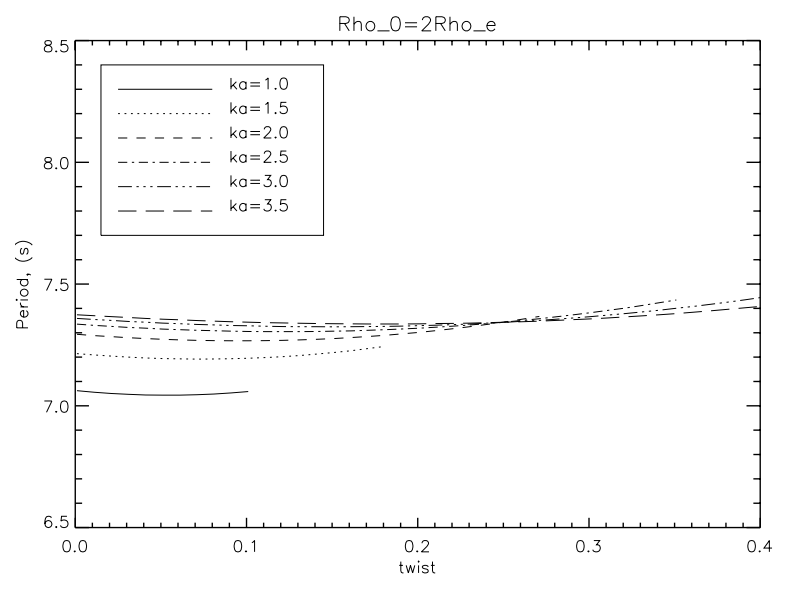

Fig. 9. This plot shows the effect of $B_{\theta}$ on the periods of oscillation for parameters the same as in Fig. 7. This plot is for the surface mode with phase speed $\approx 0.85$ at $k a=4$.
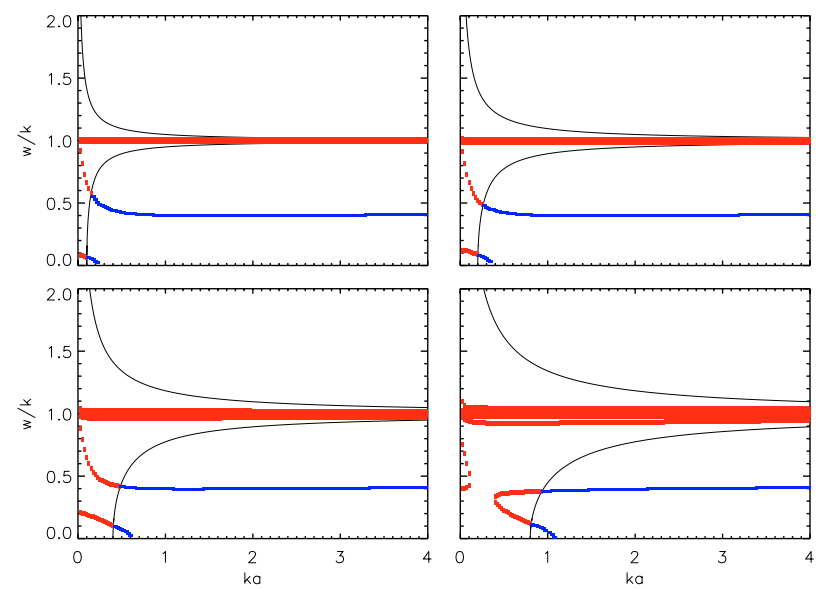

Fig. 10. Same as Fig. 3 but with parameters approximating coronal conditions. $B_{\mathrm{i}}=B_{0}, B_{\mathrm{e}}=0.5 B_{0}, a / R=0.80, \rho_{0} / \rho_{\mathrm{i}}=1, \rho_{0} / \rho_{\mathrm{e}}=0.5$. Shown are plots for twist $B_{\theta} / B_{z}=0.05,0.1,0.2,0.4$.

tube by considering an internal and annulus density half that of in the external region. We have plotted the case of an internal and annulus longitudinal magnetic field strength double the external magnetic field. Broadening of the infinite band of hybrid modes is also seen in this case. Similar to the photospheric case the avoided crossing is, again, a feature and is clearly visible in Fig. 10. The relationship between twist and period is plotted in Fig. 12 for different values of $k a$ for the surface mode in Fig. 10. Increasing the twist has a much more pronounced effect on the period for longer wavelengths. For $k a=1$, an increase in twist from 0 to 0.2 results in $\approx 1 \%$ increase in period whereas for $k a=3$ the same increase in twist sees no change in the period. Another feature of the pseudo-mode (one that changes from hybrid to purely surface) in Fig. 10 is that it has, for twists less than 0.2 , a phase speed minimum occurring at $k a \approx 1.5$ shown by the crossing of lines in Fig. 12.

\section{Asymptotic analysis}

It is of interest to now consider the cases of large and small $k_{z} a$, $k_{z} R$ corresponding to the short and long wavelength approximations. This allows us to have a first insight into oscillations and MHD waves in a twisted core-shell problem. In addition, we investigate what happens to the solutions when the annulus has small twist and when the annulus is comparatively thin. 

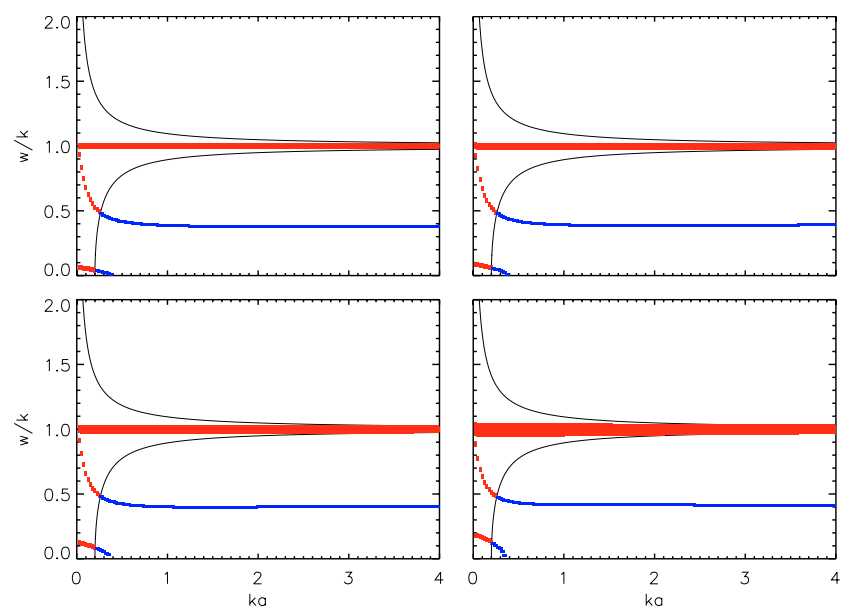

Fig. 11. Same as Fig. 10 but with fixed twist $B_{\theta} / B_{z}=0.1$. Shown are plots for annulus width $5 \%, 10 \%, 20 \%$ and $40 \%$ of the tubes radius.

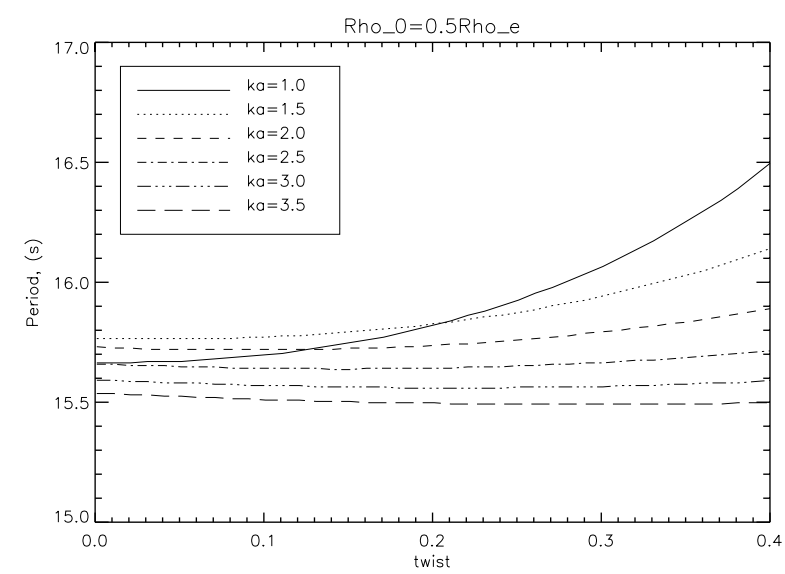

Fig. 12. This plot shows the effect of $B_{\theta}$ on the periods of oscillation for parameters the same as in Fig. 10.

To analytically describe the behaviour of the waves for the long and short wavelength approximations we restrict attention to the sausage $(m=0)$ modes. The kink $(m=1)$ and flute $(m>1)$ modes are subject to follow up investigations.

\subsection{Long wavelength $\left(k_{z} a<k_{z} R \ll 1\right)$}

We introduce the following notation:

$$
\begin{array}{ll}
c_{\mathrm{A} z}=\frac{B_{0}}{\sqrt{\mu \rho_{0}}}, & c_{a \theta}=\frac{A_{0} a}{\sqrt{\mu \rho_{0}}}, \\
c_{R \theta}=\frac{A_{0} R}{\sqrt{\mu \rho_{0}}}, & c_{\mathrm{ph}}=\frac{\omega}{k_{z}} .
\end{array}
$$

So that $c_{\mathrm{A} z}$ is the Alfvén speed due to the longitudinal component of the annulus' magnetic field, $c_{a \theta}, c_{R \theta}$ are due to the azimuthal component at each of the boundaries and $c_{\mathrm{ph}}$ is the phase speed of the modes.

\subsubsection{Purely surface modes}

For the long wavelength approximation, since

$m_{0}^{2} a^{2}=k_{z}^{2} a^{2}-\frac{4 c_{\mathrm{A} z}^{2} c_{a \theta}^{2}}{\left(c_{\mathrm{ph}}^{2}-c_{\mathrm{A} z}^{2}\right)^{2}}$, $m_{0}^{2} R^{2}=k_{z}^{2} R^{2}-\frac{4 c_{\mathrm{A} z}^{2} c_{R \theta}^{2}}{\left(c_{\mathrm{ph}}^{2}-c_{\mathrm{A} z}^{2}\right)^{2}}$,

we find that the purely surface modes (when $m_{0}^{2}>0$ ) occur when

$c_{\mathrm{ph}}>\sqrt{c_{\mathrm{A} z}^{2}+\frac{2 c_{\mathrm{A} z} c_{R \theta}}{k_{z} R}}$

and when

$c_{\mathrm{ph}}<\sqrt{c_{\mathrm{A} z}^{2}-\frac{2 c_{\mathrm{A} z} c_{R \theta}}{k_{z} R}}$.

Letting $k_{z} a<k_{z} R \ll 1$, and realising the fact that $4 c_{\mathrm{Az}}^{2} c_{R \theta}^{2} /\left(c_{\mathrm{ph}}^{2}-\right.$ $\left.c_{\mathrm{A} z}^{2}\right)^{2}<k_{z}^{2} R^{2} \ll 1$ for this case, the dispersion relation (28a) describing purely surface waves reduces to:

$\frac{K_{0}^{\prime}\left(m_{0} a\right)}{I_{0}^{\prime}\left(m_{0} a\right)}=\frac{K_{0}^{\prime}\left(m_{0} R\right)}{I_{0}^{\prime}\left(m_{0} R\right)}$

which can be re-written as

$\frac{K_{1}\left(\frac{a}{R}\left(m_{0} R\right)\right)}{I_{1}\left(\frac{a}{R}\left(m_{0} R\right)\right)}=\frac{K_{1}\left(m_{0} R\right)}{I_{1}\left(m_{0} R\right)}$,

a transcendental equation determining $m_{0} R$, and, ultimately the phase speed, $c_{\mathrm{ph}}$, and the eigenfrequency, $\omega$, for a given wavenumber, $k_{z}$. It is left to determine $c_{\mathrm{ph}}$ from

$c_{\mathrm{ph}}^{2}\left(m_{0} R\right)=c_{\mathrm{A} z}^{2}\left(1 \pm \frac{2 c_{R \theta} / c_{\mathrm{A} z}}{\sqrt{k_{z}^{2} R^{2}-\left(m_{0} R\right)^{2}}}\right)$,

re-written from Eq. (31). Relation (35), is satisfied, and becomes an identity, when $a=R$. However, for the long wavelength approximation, purely surface modes only occur, from Eqs. (32), (33), at phase speeds $c_{\mathrm{ph}} \rightarrow \pm \infty$.

\subsubsection{Hybrid modes}

Considering the hybrid modes we find that $m_{0}^{2}<0$ when

$\sqrt{c_{\mathrm{A} z}^{2}-\frac{2 c_{\mathrm{A} z} c_{a \theta}}{k_{z} a}}<c_{\mathrm{ph}}<\sqrt{c_{\mathrm{A} z}^{2}+\frac{2 c_{\mathrm{A} z} c_{a \theta}}{k_{z} a}}$,

and we write $n_{0} a=x, n_{0} R=y$ where $n_{0}^{2}=-m_{0}^{2}$. Letting $k_{z} a<k_{z} R \ll 1$, the dispersion relation (28b) for hybrid modes reduces to:

$\frac{Y_{0}(x)}{J_{0}(x)} \frac{1+\frac{c_{a \theta}}{2 c_{\mathrm{A} z}} \frac{Y_{0}^{\prime}(x)}{Y_{0}(x)}}{1+\frac{c_{a \theta}}{2 c_{\mathrm{Az}}} \frac{J_{0}^{\prime}(x)}{J_{0}(x)}}=\frac{1+\frac{c_{R \theta}}{2 c_{\mathrm{A} z}} \frac{Y_{0}^{\prime}(y)}{Y_{0}(y)}}{1+\frac{c_{R \theta}}{2 c_{\mathrm{A} z}} \frac{J_{0}^{\prime}(y)}{J_{0}(y)}} \frac{Y_{0}(y)}{J_{0}(y)}$.

Solutions of Eq. (36) have been plotted for $a / R=0.95$, Fig. 13, and for $a / R=0.71$, Fig. 14 , each for twist, $B_{\theta} / B_{0}$, values of 0.01 , $0.05,0.1$ and 0.2 . Although the $x$-axis values are from $0-4$, it should be noted that these plots, as with Figs. 15 and 16, are for the long wavelength approximation so valid for only smaller values of $k a$.

If twist is small then

$\frac{c_{a \theta}}{c_{\mathrm{A} z}}, \quad \frac{c_{R \theta}}{c_{\mathrm{A} z}} \ll 1$, 

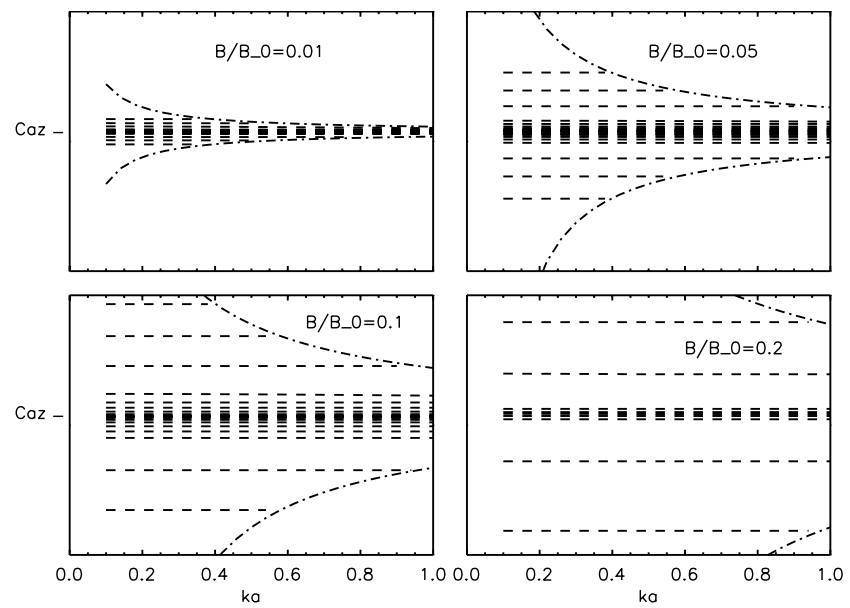

Fig. 13. Phase velocity of the $m=0$ modes determined from Eq. (36) for $a / R=0.95$ showing the considerable widening of the band of infinite hybrid modes as twist is increased. Also shown is the envelope (dotdash line) separating hybrid (dashed) modes and surface modes.
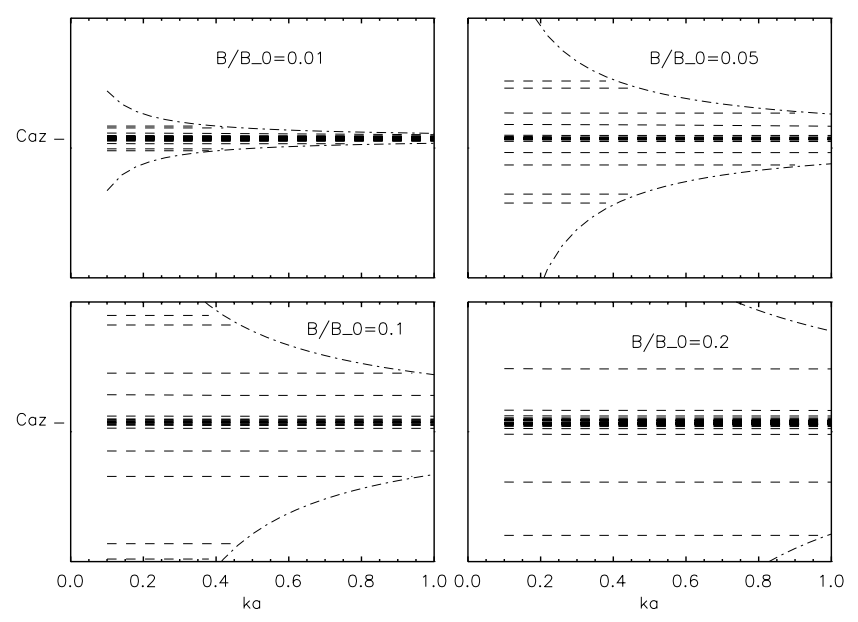

Fig. 14. Phase velocity of the $m=0$ modes determined from Eq. (36) for $a / R=0.71$ showing the considerable widening of the band of infinite hybrid modes as twist is increased. Also shown is the envelope (dotdash line) separating hybrid (dashed) modes and surface modes.

and it follows from Eq. (36) that

$$
\begin{gathered}
1+\frac{c_{a \theta}}{2 c_{\mathrm{A} z}}\left(\frac{Y_{1}(x)}{Y_{0}(x)}-\frac{J_{1}(x)}{J_{0}(x)}\right)-\frac{c_{R \theta}}{2 c_{\mathrm{A} z}}\left(\frac{Y_{1}(y)}{Y_{0}(y)}-\frac{J_{1}(y)}{J_{0}(y)}\right)= \\
\frac{Y_{0}(x)}{J_{0}(x)} \frac{J_{0}(y)}{Y_{0}(y)} .
\end{gathered}
$$

After expansion of the Bessel functions $J_{0}, J_{1}, Y_{1}$ and noting that in the long wavelength limit for small twist $x, y \ll 1$, Eq. (37) becomes

$$
1-\frac{1}{\pi c_{\mathrm{A} z}}\left(\frac{c_{a \theta}}{x Y_{0}(x)}-\frac{c_{R \theta}}{y Y_{0}(y)}\right)=\frac{Y_{0}(x)}{Y_{0}(y)},
$$

and, since $y=R x / a$ and $c_{R \theta}=R c_{a \theta} / a$ we obtain

$$
x Y_{0}(x)=\frac{c_{a \theta}}{\pi c_{\mathrm{A} z}}
$$

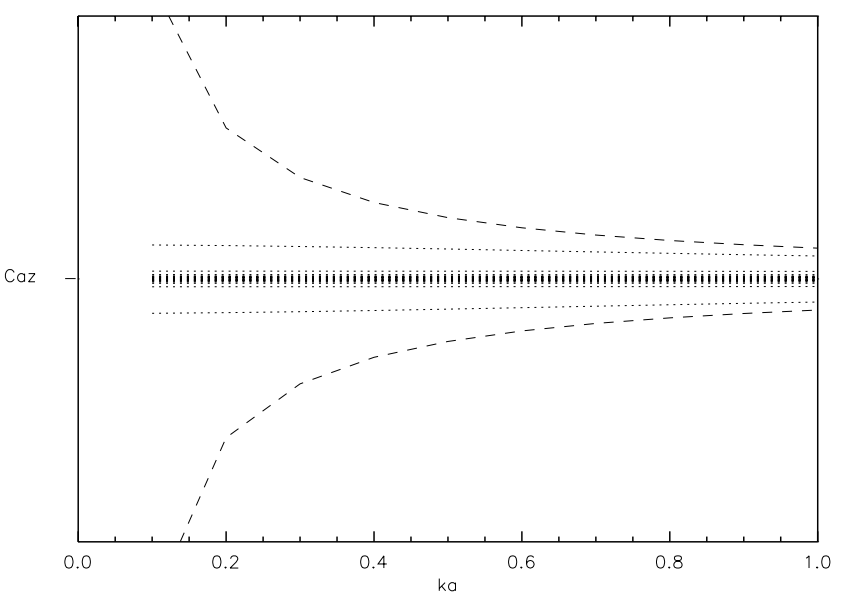

Fig. 15. Phase velocity of the $m=0$ modes determined from Eq. (39) for $B_{\theta}(a)=0.01 B_{0}$. Also shown is the envelope (dashed line) separating hybrid (dotted) modes and surface modes.

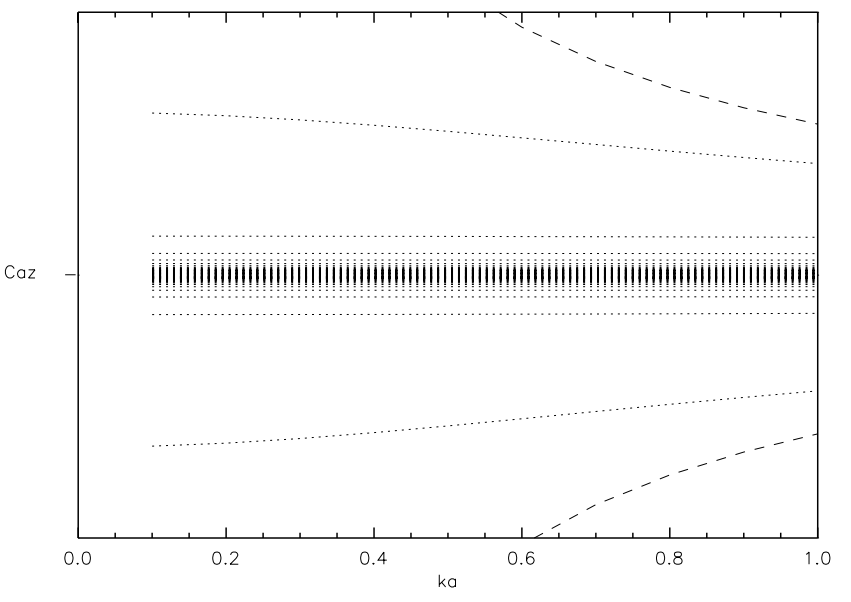

Fig. 16. Phase velocity of the $m=0$ modes determined from Eq. (39) for $B_{\theta}(a)=0.05 B_{0}$. Also shown is the envelope (dashed line) separating hybrid (dotted) modes and surface modes.

a transcendental equation determining $x$ and ultimately the phase speed $c_{\mathrm{ph}}$. We are now, for the solutions to Eq. (39), left to determine $c_{\mathrm{ph}}$ from

$c_{\mathrm{ph}}^{2}(x)=c_{\mathrm{A} z}^{2}\left(1 \pm \frac{2 c_{a \theta} / c_{\mathrm{A} z}}{\sqrt{x^{2}+k_{z}^{2} a^{2}}}\right)$.

Solutions to Eq. (39) have been plotted for two different degrees of twist (see Figs. 15, 16). As twist is increased, the infinite band of hybrid modes broadens.

\subsection{Short wavelength $(k R>k a \gg 1)$}

For the short wavelength limit, $1 \ll k a<k R$, we introduce the following notation:

$m_{0} a=x, \quad m_{0} R=y, \quad \varphi_{0}=\frac{K_{0}\left(k_{z} a\right)}{I_{0}\left(k_{z} a\right)} \frac{I_{0}\left(k_{z} R\right)}{K_{0}\left(k_{z} R\right)}$.

We notice, from Eq. (30), that $m_{0}^{2}$, for the short wavelength approximation, is greater than 0 unless $c_{\mathrm{ph}} \approx c_{\mathrm{A} z}$ so hybrid modes will occur with phase speeds $c_{\text {ph }}$ only in a close neighbourhood of $c_{\mathrm{A} z}$. We now consider the purely surface modes so that $m_{0}^{2}>0$. 
After some algebra we find from Eq. (28a) for short wavelengths that

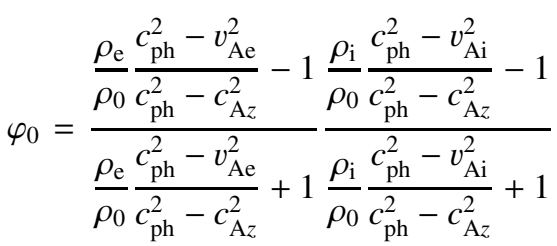

$$
\begin{aligned}
& \times\left(1-\frac{1}{x} \frac{\left(\frac{\rho_{\mathrm{i}}}{\rho_{0}}\right)^{2}-\frac{\rho_{\mathrm{i}}}{\rho_{0}} \frac{c_{\mathrm{ph}}^{2}-v_{\mathrm{Ai}}^{2}}{c_{\mathrm{ph}}^{2}-c_{\mathrm{A} z}^{2}}-2 \frac{c_{a \theta}}{c_{\mathrm{ph}}^{2}-c_{\mathrm{A} z}^{2}}}{\left(\frac{\rho_{\mathrm{i}}}{\rho_{0}}\right)^{2}\left(\frac{c_{\mathrm{ph}}^{2}-v_{\mathrm{Ai}}^{2}}{c_{\mathrm{ph}}^{2}-c_{\mathrm{A} z}^{2}}\right)-1}\right. \\
& \left.+\frac{1}{y} \frac{\left(\frac{\rho_{\mathrm{e}}}{\rho_{0}}\right)^{2}-\frac{\rho_{\mathrm{e}}}{\rho_{0}} \frac{c_{\mathrm{ph}}^{2}-v_{\mathrm{Ae}}^{2}}{c_{\mathrm{ph}}^{2}-c_{\mathrm{A} z}^{2}}-2 \frac{c_{R \theta}}{c_{\mathrm{ph}}^{2}-c_{\mathrm{A} z}^{2}}}{\left(\frac{\rho_{\mathrm{e}}}{\rho_{0}}\right)^{2}\left(\frac{c_{\mathrm{ph}}^{2}-v_{\mathrm{Ae}}^{2}}{c_{\mathrm{ph}}^{2}-c_{\mathrm{A} z}^{2}}\right)-1}\right) .
\end{aligned}
$$

This is of too high order in $c_{\mathrm{ph}}$ to be analytically solvable for the eigenfrequency, $\omega$. However, it is possible to further simplify for the slender annulus $((R-a) / R \ll 1)$ limit. We are able to re-write the LHS of Eq. (40) in the following way:

$\varphi_{0} \approx \mathrm{e}^{2 m_{0}(R-a)}\left(1-\frac{R-a}{4 m_{0} a R}\right)$

Thus in the slender annulus limit $(R-a) / R \ll 1$ we find:

$\varphi_{0} \approx 1+2(R-a) m_{0}$.

Using the fact that $x, y \gg 1$ (short wavelength) and $(R-a) / R \ll$ 1 (slender annulus), Eq. (40) can be reduced to give

$$
\begin{gathered}
\left(\frac{\rho_{\mathrm{e}}}{\rho_{0}} \frac{c_{\mathrm{ph}}^{2}-v_{\mathrm{Ae}}^{2}}{c_{\mathrm{ph}}^{2}-c_{\mathrm{A} z}^{2}}+1\right)\left(\frac{\rho_{\mathrm{i}}}{\rho_{0}} \frac{c_{\mathrm{ph}}^{2}-v_{\mathrm{Ai}}^{2}}{c_{\mathrm{ph}}^{2}-c_{\mathrm{A} z}^{2}}+1\right)= \\
\left(\frac{\rho_{\mathrm{e}}}{\rho_{0}} \frac{c_{\mathrm{ph}}^{2}-v_{\mathrm{Ae}}^{2}}{c_{\mathrm{ph}}^{2}-c_{\mathrm{A} z}^{2}}-1\right)\left(\frac{\rho_{\mathrm{i}}}{\rho_{0}} \frac{c_{\mathrm{ph}}^{2}-v_{\mathrm{Ai}}^{2}}{c_{\mathrm{ph}}^{2}-c_{\mathrm{A} z}^{2}}-1\right)
\end{gathered}
$$

which yields the solutions

$$
c_{\mathrm{ph}}^{2}=c_{\mathrm{A} z}^{2}, \quad c_{\mathrm{ph}}^{2}=\frac{\rho_{\mathrm{i}} v_{\mathrm{Ai}}^{2}+\rho_{\mathrm{e}} v_{\mathrm{Ae}}^{2}}{\rho_{\mathrm{i}}+\rho_{\mathrm{e}}}=c_{k}^{2},
$$

a similar result to that found by Bennett et al. (1999). These two modes are the surface wave (as obtained in Cartesian geometry such as the magnetic surface and slab by e.g. Roberts 1981a,b) and body waves of the system. The first solution in Eq. (44) are the trapped Alfvén waves which, in the limit of thin annulus, all become the same.

\section{Conclusions}

We considered an incompressible medium and, to study the role of the magnetic field, gravity effects are ignored. For a magnetic configuration consisting of an internal region, annulus and external ambient region each with uniform yet distinct twist the full analytical dispersion relation was obtained. More specifically, we analysed the case where the internal and external regions have only magnetic field in the $z$ direction (in a cylindrical coordinate system), only the annulus having a uniform twist. Again, the analytical dispersion relation was found and we investigated numerically for the cylindrically symmetric $m=0$ (sausage) mode the existence and behaviour of the different modes for a wide range of typical solar atmospheric parameter values. We also investigated analytically the asymptotic cases of long and short wavelength approximation. We considered the case of photospheric tubes, present in, e.g. sunspots, plage regions, largescale unipolar areas and supergranulation fields at supergranule boundaries; coronal loops as seen by TRACE, and finally in evacuated tubes. In each case we solved the appropriate dispersion relation and plotted the dispersion diagrams for varying twist and varying annulus thickness in the order to determine the role of each of these characteristics. It was found, in each case, that the solutions consisted of an infinite band of hybrid modes centered around the Alfvén speed due to the longitudinal component of magnetic field in the annulus, $c_{\mathrm{A} z}$. In the incompressible case, when there is no applied twist, there are no hybrid modes present (see Bennett et al. 1999; Edwin \& Roberts 1983), as twist is introduced the hybrid modes begin to occur. It was found that as twist is increased, the infinite band of hybrid modes broadens and as the applied twist becomes large, possible indications of instabilities (shown as the avoided crossing feature) appear. It was also apparent that an increase in the annulus thickness (in comparison to the tube thickness) had a similar effect of broadening the infinite band of modes and eventual destabalising effects on slower modes.

The long wavelength approximation analysis confirmed these findings by also showing a widening of the infinite band of hybrid modes. The additional assumption of small twist also clearly shows the widening of the infinite band. In the short wavelength approximation it was analytically shown that, for hybrid modes, as $k a \rightarrow \infty$ then the phase speed $c_{\mathrm{ph}} \rightarrow c_{\mathrm{Az} z}$. For surface modes, including the assumption of slender annulus, we found solutions were tending to the tube kink speed, $c_{k}$, where $c_{k}^{2}=\left(\rho_{\mathrm{i}} v_{\mathrm{Ai}}^{2}+\rho_{\mathrm{e}} v_{\mathrm{Ae}}^{2}\right) /\left(\rho_{\mathrm{i}}+\rho_{\mathrm{e}}\right)$.

For photospheric values, taking e.g. $k a=1$, we found that increasing the twist from $B_{\theta} / B_{z}=0.001$ to $B_{\theta} / B_{z}=0.1$ caused an increase in period of $\approx 1 \%$, and a further increase of twist, to 0.2 , resulted in $\approx 2 \%$ increase in the period (see Fig. 5). Also investigated was the effect that the strength of the magnetic field in the tube has on the period. Figure 6 shows that for larger values of $k a$, the increase in $B_{0} / B_{\mathrm{e}}$ (the ratio of internal magnetic field strength to external), has the same effect on the period (namely an increase of $\approx 0.5 \%$ for Fig. 6) regardless of the amount of applied twist. However, for longer wavelengths (when $k a<\approx 1$ ), the value of the twist has an effect on how much increasing the internal magnetic field strength increases the period.

For coronal values, the effect of twist is less pronounced. The value of $k a$ where the pseudo-mode (one that changes from hybrid to purely surface) shown in Figs. 7-8 changes from hybrid to purely surface is highly dependant on the twist. For large values of $k a$ the period of the surface mode is relatively unaltered by the amount of twist. For smaller $k a$ values the surface mode only exists for smaller amounts of twist. Evacuated tube parameters, modelled as an internal density half that of the external density, were analysed. Figure 12 shows the importance of twist on the periods of oscillation (for the surface mode at $c_{\mathrm{ph}} \approx 0.4$ ). For $k a=1$, an increase of twist from 0.001 to 0.1 results in an increase in period of $\approx 0.5 \%$, and increasing the twist to 0.2 gives a further increase of $0.8 \%$. Further study will hopefully lead to examining the case of a twisted annulus without the discontinuity in magnetic field at the inner annulus boundary in order to extend understanding of heating processes within the solar atmosphere. 
Acknowledgements. The authors thank Prof. M. Ruderman and N. Venkov for a number of useful discussions. R.E. acknowledges M. Kéray for patient encouragement. The authors are also grateful to NSF, Hungary (OTKA, Ref. No TO43741) and to The University of Sheffield (White Rose Consortium) for the financial support they received.

\section{References}

Appert, K., Gruber, R., \& Vaclavik, J. 1974, Phys. Fluids, 17, 1471

Aschwanden, M. J. 2003, Review of coronal oscillations, in Turbulence, Waves and Instabilities in the Solar Plasma, ed. Erdélyi, et al., NATO Sci Ser., 124, 215

Ashwanden, M. J. 2004, Physics of the Solar Corona (Chichester, UK: Praxis Publishing Ltd.)

Aschwanden, M. J., Fletcher, L., Schrijver, C. J., et al. 1999, ApJ, 520, 880

Aschwanden, M. J., Nightingale, R.W., Andries, J., et al. 2003, ApJ, 598, 1375

Bennett, K., Roberts, B., \& Narain, U. 1999, Sol. Phys., 185, 41

Bogdan, T. J. 1984, ApJ, 282, 769.

Carter, B. K. 2005, in Plasma and Astrophysics: from laboratory to outer space, ed. I. Ballai, et al., PADAU, 15, 115

Carter, B. K., \& Erdélyi, R. 2004, in Coronal Heating, ESA SP-575, ed. R. W. Walsh, et al., 378

Chae, J., Wang, H., Qiu, J., et al. 2000, ApJ, 533, 535

de Pontieu, B., Erdélyi, R., \& James, S. P. 2004, Nature, 430, 536

de Pontieu, B., Erdélyi, R., \& De Moortel, I. 2005, ApJ, 624, 61

Dungey, J. W., \& Loughhead, R. E. 1954, Austr. J. Phys., 7, 5

Edwin, P. M., \& Roberts, B. 1982, Sol. Phys., 76, 239
Edwin, P. M., \& Roberts, B. 1983, Sol. Phys., 88, 179

Erdélyi, R. 2006, Phil. Trans. Roy. Soc., A, 364, 351

Goedbloed, J. P. 1971a, Physica, 53, 412

Goedbloed, J. P. 1971b, Physica, 53, 501

Goedbloed, J. P. 1971c, Physica, 53, 535

Goedbloed, J. P. 1983, Lecture Notes on Ideal Magnetohydrodynamics, Rijnhuizen Rep., 83

Goossens, M., Ruderman, M. S., \& Hollweg, J. V. 1995, Sol. Phys., 145, 19

Goossens, M., Andries, J., \& Aschwanden, M. J. 2002, A\&A, 394, 39

Hain, K., \& Lüst, R. 1958, Z. Naturforsch, 13a, 936

Klimchuk, J. A., Antiochos, S. K., \& Norton, D. 2000, ApJ, 542, 504

Mikhalyaev, B. B., \& Solov'ev, A. A. 2005, Sol. Phys., 227, 249

Nakariakov, V. M., \& Roberts, B. 2003, in Turbulence, Waves and Instabilities in the Solar Plasma, ed. Erdélyi, et al., NATO Sci Ser., 124, 167

Nakariakov, V. M., Ofman, L., Deluca, E. E., et al. 1999, Science, 285, 862

Ofman, L., \& Aschwanden, M. J. 2002, ApJ, 576, 153

Parker, E. N. 1974, ApJ, 191, 245

Roberts, B. 1981a, Sol. Phys., 69, 27

Roberts, B. 1981b, Sol. Phys., 69, 39

Roberts, B. 2000, Sol. Phys., 193, 139

Roberts, B., Edwin, P. M., \& Benz, A. O. 1984, ApJ, 279, 857

Ruderman, M. S., \& Roberts, B. 2002, ApJ, 577, 475

Stenuit, H., Keppens, R., \& Goossens, M. 1998, A\&A, 331, 392

Stenuit, H., Tirry, W. J., Keppens, R., et al. 1999, A\&A, 342, 863

Van Doorsselaere, T., Andries, J., Poedts, S., et al. 2004, ApJ, 606, 1223

Wang, T. J. 2004, in Waves, oscillations and small scale transient events in the solar atmosphere: A joint view of SOHO and TRACE, ed. R., Erdélyi, et al., ESA SP-547, 417 
R. Erdélyi and B. K. Carter: Wave propagation in twisted magnetic Annulus, Online Material p 1

\section{Online Material}




\section{Appendix A:}

The general dispersion relation for a magnetic core, annulus and external field all with uniform, though distinct for each region, twist given by

$\boldsymbol{B}= \begin{cases}\boldsymbol{B}_{\mathrm{i}}=\left(0, A_{\mathrm{i}} r, B_{\mathrm{i}}\right), & r<a, \\ \boldsymbol{B}_{0}=\left(0, A_{0} r, B_{0}\right), & a<r<R, \\ \boldsymbol{B}_{\mathrm{e}}=\left(0, A_{\mathrm{e}} r, B_{\mathrm{e}}\right), & r>R,\end{cases}$

is found:

$$
\begin{gathered}
\frac{\Xi_{a K}-\Xi_{\mathrm{i}}+\Xi_{a K} \Xi_{\mathrm{i}} \frac{A_{0}^{2}-A_{\mathrm{i}}^{2}}{\mu}}{\Xi_{a I}-\Xi_{\mathrm{i}}+\Xi_{a I} \Xi_{\mathrm{i}} \frac{A_{0}^{2}-A_{\mathrm{i}}^{2}}{\mu}} \frac{K_{m}\left(m_{0} a\right)}{I_{m}\left(m_{0} a\right)}=\frac{K_{m}\left(m_{0} R\right)}{I_{m}\left(m_{0} R\right)} \frac{\Xi_{R K}-\Xi_{\mathrm{e}}+\Xi_{R K} \Xi_{\mathrm{e}} \frac{A_{0}^{2}-A_{\mathrm{e}}^{2}}{\mu}}{\Xi_{R I}-\Xi_{\mathrm{e}}+\Xi_{R I} \Xi_{\mathrm{e}} \frac{A_{0}^{2}-A_{\mathrm{e}}^{2}}{\mu}}, \quad m_{0}^{2}>0, \\
\text { for purely surface waves, } \\
\frac{\Xi_{a Y}-\Xi_{\mathrm{i}}+\Xi_{a Y} \Xi_{\mathrm{i}} \frac{A_{0}^{2}-A_{\mathrm{i}}^{2}}{\mu}}{\Xi_{a J}-\Xi_{\mathrm{i}}+\Xi_{a J} \Xi_{\mathrm{i}} \frac{A_{0}^{2}-A_{\mathrm{i}}^{2}}{\mu}} \frac{Y_{m}\left(m_{0} a\right)}{J_{m}\left(m_{0} a\right)}=\frac{Y_{m}\left(m_{0} R\right)}{J_{m}\left(m_{0} R\right)} \frac{\Xi_{R Y}-\Xi_{\mathrm{e}}+\Xi_{R Y} \Xi_{\mathrm{e}} \frac{A_{0}^{2}-A_{\mathrm{e}}^{2}}{\mu}}{\Xi_{R J}-\Xi_{\mathrm{e}}+\Xi_{R J} \Xi_{\mathrm{e}} \frac{A_{0}^{2}-A_{\mathrm{e}}^{2}}{\mu}}, \quad m_{0}^{2}<0,
\end{gathered}
$$

for hybrid (body-surface) waves.

Here

$\Xi_{\alpha X}=\frac{\left(\omega^{2}-\omega_{\mathrm{A} 0}^{2}\right) \frac{m_{0} \alpha X_{m}^{\prime}\left(m_{0} \alpha\right)}{X_{m}\left(m_{0} \alpha\right)}-\frac{2 m A_{0} \omega_{\mathrm{A} 0}}{\sqrt{\mu \rho_{0}}}}{\rho_{0}\left(\omega^{2}-\omega_{\mathrm{A} 0}^{2}\right)^{2}-\frac{4 A_{0}^{2} \omega_{\mathrm{A} 0}^{2}}{\mu}}$,

where $X$ denotes the corresponding Bessel function and $\alpha$ is replaced by $a, R$ for the corresponding region,

$$
\begin{gathered}
\Xi_{\mathrm{i}}=\frac{\left(\omega^{2}-\omega_{\mathrm{Ai}}^{2}\right) \frac{m_{\mathrm{a}} I_{m}^{\prime}\left(m_{\mathrm{i}} a\right)}{I_{m}\left(m_{\mathrm{i}} a\right)}-\frac{2 m A_{\mathrm{i}} \omega_{\mathrm{Ai}}}{\sqrt{\mu \rho_{\mathrm{i}}}}}{\rho_{\mathrm{i}}\left(\omega^{2}-\omega_{\mathrm{Ai}}^{2}\right)^{2}-\frac{4 A_{\mathrm{i}}^{2} \omega_{\mathrm{Ai}}}{\mu}}, \quad \Xi_{\mathrm{e}}=\frac{\left(\omega^{2}-\omega_{\mathrm{Ae}}^{2}\right) \frac{m_{\mathrm{e}} R K_{m}^{\prime}\left(m_{\mathrm{e}} R\right)}{K_{m}\left(m_{\mathrm{e}} R\right)}-\frac{2 m A_{\mathrm{e}} \omega_{\mathrm{Ae}}}{\sqrt{\mu \rho_{\mathrm{e}}}}}{\rho_{\mathrm{e}}\left(\omega^{2}-\omega_{\mathrm{Ae}}^{2}\right)^{2}-\frac{4 A_{\mathrm{e}}^{2} \omega_{\mathrm{Ae}}^{2}}{\mu}}, \\
m_{\mathrm{i}}^{2}=k_{z}^{2}\left(1-\frac{4 A_{\mathrm{i}}^{2} \omega_{\mathrm{Ai}}^{2}}{\mu \rho_{\mathrm{i}}\left(\omega^{2}-\omega_{\mathrm{Ai}}^{2}\right)^{2}}\right), \quad m_{0}^{2}=k_{z}^{2}\left(1-\frac{4 A_{0}^{2} \omega_{\mathrm{A} 0}^{2}}{\mu \rho_{0}\left(\omega^{2}-\omega_{\mathrm{A} 0}^{2}\right)^{2}}\right), \quad m_{\mathrm{e}}^{2}=k_{z}^{2}\left(1-\frac{4 A_{\mathrm{e}}^{2} \omega_{\mathrm{Ae}}^{2}}{\mu \rho_{\mathrm{e}}\left(\omega^{2}-\omega_{\mathrm{Ae}}^{2}\right)^{2}}\right),
\end{gathered}
$$

where the dash ' denotes the derivative with respect to the argument of the Bessel functions. The Alfvén speeds in the annulus, internal and external regions are given by $\omega_{\mathrm{A} 0}, \omega_{\mathrm{Ai}}$, and $\omega_{\mathrm{Ae}}$, respectively such that

$\omega_{\mathrm{A} 0}=\frac{1}{\sqrt{\mu \rho_{0}}}\left(m A_{0}+k_{z} B_{0}\right), \quad \omega_{\mathrm{Ai}}=\frac{1}{\sqrt{\mu \rho_{\mathrm{i}}}}\left(m A_{\mathrm{i}}+k_{z} B_{\mathrm{i}}\right), \quad \omega_{\mathrm{Ae}}=\frac{1}{\sqrt{\mu \rho_{\mathrm{e}}}}\left(m A_{\mathrm{e}}+k_{z} B_{\mathrm{e}}\right)$.

By letting $A_{\mathrm{i}}=0$ and $A_{\mathrm{e}}=0$, so that we now consider the case of a straight internal and external field with a twisted annulus, Eqs. (A.6) reduce to:

$m_{\mathrm{i}}^{2}=k_{z}^{2}, \quad m_{\mathrm{e}}^{2}=k_{z}^{2}, \quad m_{0}^{2}=k_{z}^{2}\left(1-\frac{4 A_{0}^{2} \omega_{\mathrm{A} 0}^{2}}{\mu \rho_{0}\left(\omega^{2}-\omega_{\mathrm{A} 0}^{2}\right)^{2}}\right)$,

so that $m_{\mathrm{i}} a=\left|k_{z}\right| a$ and $m_{\mathrm{e}} R=\left|k_{z}\right| R$ which are always positive. We can now rewrite the definitions (A.5) as

$\Xi_{\mathrm{i}}=\frac{|k| a I_{m}^{\prime}(|k| a)}{\rho_{\mathrm{i}}\left(\omega^{2}-\omega_{\mathrm{Ai}}^{2}\right) I_{m}(|k| a)}, \quad \Xi_{\mathrm{e}}=\frac{|k| R K_{m}^{\prime}(|k| R)}{\rho_{\mathrm{e}}\left(\omega^{2}-\omega_{\mathrm{Ae}}^{2}\right) K_{m}(|k| R)}$,

so that we recover the general dispersion relations for a twisted annulus embedded in a straight internal and external field given by Eqs. (28a), (28b). 
R. Erdélyi and B. K. Carter: Wave propagation in twisted magnetic Annulus, Online Material p 3

\section{Appendix B:}

The general dispersion relation for surface modes, Eq. (28a) can be recast as

$$
\begin{array}{r}
\frac{c_{\mathrm{A} 0} \mathcal{I}_{m}-\frac{2 m A_{0} \omega_{\mathrm{A} 0}}{\sqrt{\mu \rho_{0}}}}{c_{\mathrm{A} 0}^{2}-\frac{4 A_{0}^{2} \omega_{\mathrm{A} 0}^{2}}{\rho_{0} \mu}}-\frac{\mathcal{K}_{k}}{\frac{\rho_{\mathrm{e}}}{\rho_{0}} c_{\mathrm{Ae}}+\frac{A_{0}^{2}}{\mu \rho_{0}} \mathcal{K}_{k}}-\left(\frac{c_{\mathrm{A} 0} \mathcal{K}_{R}-\frac{2 m R \omega_{\mathrm{A} 0}}{\sqrt{\mu \rho_{0}}}}{c_{\mathrm{A} 0}^{2}-\frac{4 A_{0}^{2} \omega_{\mathrm{A} 0}^{2}}{\mu \rho_{0}}}-\frac{\mathcal{K}_{k}}{\frac{\rho_{\mathrm{e}}}{\rho_{0}} c_{\mathrm{Ae}}+\frac{A_{0}^{2}}{\mu \rho_{0}} \mathcal{K}_{k}}\right) \\
\times\left\{\frac{\frac{c_{\mathrm{A} 0} \mathcal{I}_{a}-\frac{2 m A_{0}^{2} \omega_{\mathrm{A} 0}^{2}}{\sqrt{\mu \rho_{0}}}}{c_{\mathrm{A} 0}^{2}-\frac{4 A_{0}^{2} \omega_{\mathrm{A} 0}^{2}}{\mu \rho_{0}}}\left(\frac{\rho_{\mathrm{i}}}{\rho_{0}}+\frac{\mathcal{I}_{k} A_{0}^{2}}{c_{\mathrm{Ai}} \rho_{0} \mu}\right)-\frac{\mathcal{I}_{k}}{c_{\mathrm{Ai}}}}{\frac{c_{\mathrm{A} 0} \mathcal{K}_{a}-\frac{2 m A_{0}^{2} \omega_{\mathrm{A} 0}^{2}}{\sqrt{\mu \rho_{0}}}}{c_{\mathrm{A} 0}^{2}-\frac{4 A_{0}^{2} \omega_{\mathrm{A} 0}^{2}}{\mu \rho_{0}}}\left(\frac{\rho_{\mathrm{i}}}{\rho_{0}}+\frac{\mathcal{I}_{k} A_{0}^{2}}{c_{\mathrm{Ai}} \rho_{0} \mu}\right)-\frac{\mathcal{I}_{k}}{c_{\mathrm{Ai}}}} \frac{I_{m}\left(m_{0} R\right)}{K_{m}\left(m_{0} a\right) I_{m}\left(m_{0} R\right)}\right\}=0
\end{array}
$$

where

$$
\begin{aligned}
& \mathcal{I}_{a}=\frac{m_{0} \alpha I_{m}^{\prime}\left(m_{0} a\right)}{I_{m}\left(m_{0} a\right)}, \quad \mathcal{I}_{R}=\frac{m_{0} \alpha I_{m}^{\prime}\left(m_{0} R\right)}{I_{m}\left(m_{0} R\right)}, \quad \mathcal{I}_{k}=\frac{m_{0} \alpha I_{m}^{\prime}\left(\left|k_{z}\right| R\right)}{I_{m}\left(\left|k_{z}\right| R\right)}, \\
& \mathcal{K}_{a}=\frac{m_{0} \alpha K_{m}^{\prime}\left(m_{0} R\right)}{K_{m}\left(m_{0} R\right)}, \quad \mathcal{K}_{R}=\frac{m_{0} \alpha K_{m}^{\prime}\left(m_{0} R\right)}{K_{m}\left(m_{0} R\right)}, \quad \mathcal{K}_{k}=\frac{m_{0} \alpha K_{m}^{\prime}\left(\left|k_{z}\right| R\right)}{K_{m}\left(\left|k_{z}\right| R\right)}, \\
& c_{\mathrm{A} 0}=\left(\omega^{2}-\omega_{\mathrm{A} 0}^{2}\right) / k_{z}^{2}, \quad c_{\mathrm{Ai}}=\left(\omega^{2}-\omega_{\mathrm{Ai}}^{2}\right) / k_{z}^{2}, \quad c_{\mathrm{Ae}}=\left(\omega^{2}-\omega_{\mathrm{Ae}}^{2}\right) / k_{z}^{2},
\end{aligned}
$$

which can be compared to the dispersion relation for a twisted tube in a straight magnetic environment as found by Bennett et al. (1998) whose result can be seen as the first two terms in Eq. (B.1). 